\title{
CITOGENETICA DE OXALIS TUBEROSA: CICLO CELULAR Y NUMERO CROMOSOMICO
}

DAVID TALLEDO

CAROLA ESCOBAR

Laboniturio de Bidiogin Celuler y Centoica. Secoivin de Clicpentidoa. Faculnd de Ciendias Biolbgicas, Universidad Ficardo Palma. Apedo ISK, Lims-PERU.

\section{RESUMFN}

Se describe por primera vez is secuencia del ciclo celular de la oca Orabis nuberosa Mol., estableciéndose la incidencia y duracida de cada fase, asi como de toda la minosis, en base a la evaluación ciclica - para ve periodo de 24 haras - $d e$ los indices de fases e indices mitoticos parciales y totales, segün el metodo propuesto por Dyer. Se registran alteraciones del cunso del cislo celaiar, particulanmente cielos incoenpletos. Los autoges sugieren que la alternancia de interfaces normales con milosis intgulares podrta condicionar la diversidad de reportes para el nùmcro de cromosomas de la oca $y$ conscituir uno de los probables mecanismos de poliploidización somilika. Para la moestra de wOczs estadiada se plantea que $2 \mathrm{n=14.}$

PALABRAS CLAVES Dwalk, ciclo celular, poliplooidización.

\section{SUMMARY}

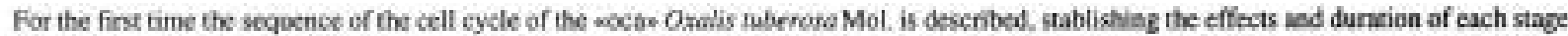
as well as of the whole mitosis based ot a cycle study-for a 24 hour period-of phase indexes and partial and tocal mitotie indeves accoeding to the method proposed by Dyer. Arerations along the cell cycle are recoeded. specially incomplete cycles. The auchors sugesests that altemance of aormal interphases with irregular mitosis may candition the varicty of reports for the namber of chronsosotnes of acese sadd become one of the probuble meshanisms of somatic polyplowdization. For the sample of acear studied a $2 \mathrm{~s}=14 \mathrm{is}$ proposed.

KEY WORDS: Omilis. cell cycle, polyploidization.

\section{INTRODUCCION.}

El género Oxalís está formado por 800 especjes aproximadamente, distribuidas en casi todos los hábitats de América del Sur y de Africa del Sur (Marks, 1956). O. tuberosa Molina *Oca» es considerada como la de mayor importancia económica entre las especies de este género. lo que se debe a su alto contenido protéico y lipidico ast como a su capacidad de tuberizar, singular entre las especies de Oxalis (Mostacero y Mejía, 1993). Los estudios realizados en oOcan hasta el momento se orientan principalmente a su descripción botánica, clasificación taxonómica y tipos de hábitat, entre otros (Brücher, 1969; Cárdenas, 1969; CIRF, 1982; Eiten, 1963; León, 1964; Gershoff, 1987). Se ha realizado así mismo una serie de estudios con la finalidad de determinar el número de cromosomas de esta especie, habiéndose obtenido resultados aparentemente contradictorios y de difícil interpretación: $2 \mathrm{n}=14$ (Heitz, 1927). $2 \mathrm{n}=63-68-70$ (Kostoff et al, 1935), $2 \mathrm{n}=66$ (Cárdenas \& Hawkes, 1948), 2n=66 (Marks, cit. de Smith. 1976), $2 \mathrm{n}=58$-66 (Gibbser al., 1978), $2 \mathrm{n}=64$ (Medina, 1994), entre otros. En cualquier caso, no se conoce con precisión cuál es el nümero de cromosomas de esta especie. Esta situación parece repetirse a nivel de todo el género (ver cuadro 3 ).

Marks (1956), Fiódorov (1969) y Brüchet (1969) reportan una gran variabilidad respecto al número, tamaño y forma de los cromosomas de las especies de este genero. Gibbs (1978) sugiere que una considerable variabilidad numérica ha sido condicionada por la formación de multivalentes y el retraso de la replicación cromosómica, esta variabilidad habría sido protegida contra la acción de la selección natural mediante la propagación clonal a través de tubérculos. El mismo autor subraya, ass mismo, que la evidenciación de cromosomas mitóticos, especialmente metafásicos, presenta dificultades. Por otro lado, se ha observado que germoplasma de oca introducido para su reproducción in vitro en algunos casos no ha mantenido las caracteristicas de la muestra original y que los métodos utilizados para evaluar su estabilidad genética han resultado insuficientes (Estrada, 1994; Velasco, 1994).

Aunque el origen de la "Ocas es poco conocido, los contajes de cromosomas demuestran que éstas son poliploides en la mayoria de los casos, lo que refleja su capacidad de adaptación a condiciones climáticas severas $y$ altas elevaciones (Del Rio, 1990).

Si bien la determinación de los datos citológicos que se derivan mayormente del núcleo celular, el comportamiento de los cromosomas durante la mitosis y/o meiosis y del estudio de su número y morfologin pueden ser usados para esclarecer la senda cvolutiva y como caracteres 
taxonómicos constantes debido a que todos los individuos de una especic usualmente presentan el mismo número de cromosomas, también es cierto que pueden presentarse excepciones (Skebbins, 1970, Turkow etal.,1988). Estas se circuscriben especialmente a los poliploides (Barlow, 1978; Brodsky \& Urivayeva, 1981; Talledo \& Escobar, 1995; ver el articulo anterior del presente número de Biotempo). La poliploidización puede producirse cuando los organismos se encuentran en condiciones de aislamiento en hábitats que presentan diferentes variaciones ambientales, como es el caso de las especies que se desarrollan en la Zona Andina (National Research Council, 1989; Jardín Botánico de Cordoba España, 1992; Talledo \& Escobar, 1995). Está demostrado que la poliploidización es una de las consecuencias posibles de la inhibición parcial de los procesos mitóticos. Por otro lado, además de poliploidía, se puede producir síntesis irregular de ADN, células multinucleadas y/o endorreproducción como resultado del acortamiento del programa del ciclo celular. La selección natural climinará o no a la progenie cuyo genoma haya variado (Brodsky \& Urivayeva, 1981. Talledo \& Escobar, 1995).

Asf mismo, la duración del ciclo celular depende el peso del genoma : cuanto mayor es la cantidad de ADN mayor es la duración del período de síntesis y del ciclo. Esta relacion, seôalada por primera vez por Van't Hoff y Sparrow en 1963, ha sido estudiada por muchos autores. El espectro de resultados para las diferentes especies es grande, pero la correlación entre el contenido de ADN en el genoma diploide y la duración del ciclo mitótico es indudable (Ivanov, 1978). Bennett (1972) sef̂ala que cuando se comparan las plantas efimeras, las anuales y las perennes, la cantidad de $A D N$ en el genoma diploide y el tiempo de reproducción de las ólulas frecuentemente forman una curva en crecimiento. Las formas diploides y poliploides de una misma especie por lo general presentan ciclos similares.

\section{MATERIALES Y METODOS.}

Los meristemos radiculares de Oxalis fuberosa Mol. (Foto 1) procedentes de tubérculos recolectados en la ciudad de Huancayo - Perú (Ref. Ing Glicerio López, Universidad Nacional del Centro del Perú) fueron procesados según la metódica descrita por Talledo et al. (1993) a intervalos de 1 hora durante 24 horas. El squash se realizó con orceína acética, tomando las microfotograflas con una pelicula en blanco y negro de baja sensibilidad marca Mikrat 300.

Los indices de fases (IF), índices mitóticos (IM) y duración del ciclo celular fueron determinados utilizando los criterios descritos en la guia propuesta por D. Talledo y C. Escobar (1995).

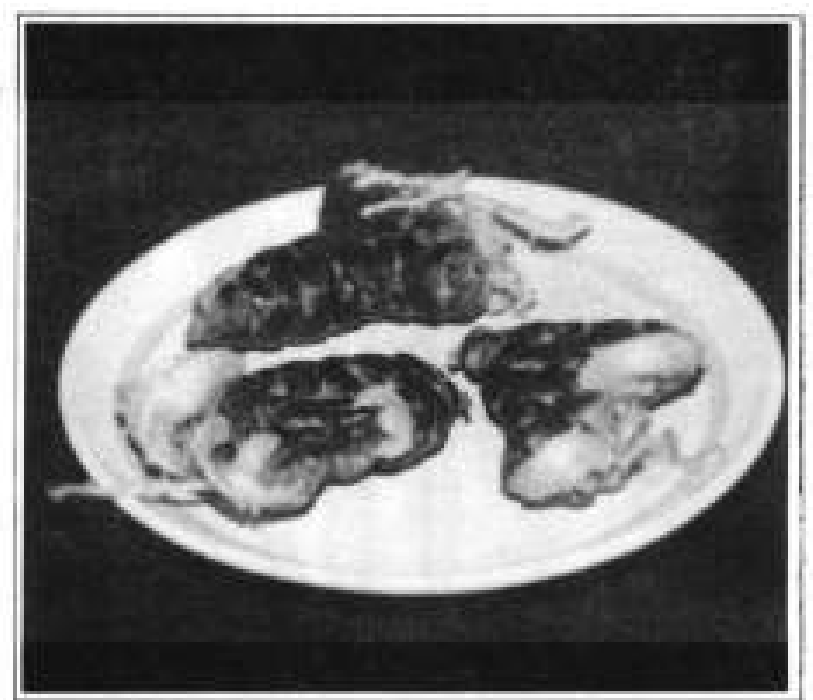

Foto 8. Accesión de Oxalis tuberosa Mol, (Huancayo, Perai)

\section{RESULTADOS}

Los cuadros 1A y 1B muestran la evaluación durante 24 horas (a una temperatura de $22^{\circ} \mathrm{C} \pm 1$ ) de los IFe IM de Oxalis ruberasa Mol. oOca en 24, 597 células en división y en las celulas hijas producidas a partir de las primeras. En ellos es posible observar que la mejor hora para la prefijación de las muestras con inhibidores de la metafase está comprendida entre las 7:30 y las 8:30 am, El valor del IM observado en este lapso es elevado y se

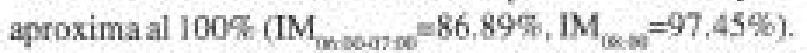
Durante las demás horas procesadas los valores del IM fueron relativamente bajos, fluctuando entre el $39.28 \%$ a las 9:00 am. y 1.59\% a las 21:00 horas. Sin embargo, los valores del IM registrados para las 23:00 horas fueron extrañamente elevados, alcanzando el $89,19 \%$.

En estas condiciones el ciclo celular parece durar 06:00 horas aproximadamente. sin embargo esto se debe a la alteración de su flujo normal, como veremos más adelante. Los valores nomnades para la duración de este ciclo son 75 minutos.

Cuando desagregamos el IM en sus componentes por fases es posible observar que entre las 6:00 y las 10:00 horas el porcentaje de profases ocupa la mayor parte de la población celular en división. Durante las demás horas, excepto las 23:00, se acumulan telofases. Las fases restantes (metafase $y$ anafase) parecen presentarse en muy pequeña proporción, o no presentarse, y su incidencia sobre el desarrollo y duración del ciclo celular es poco significativa. Al analizar el ciclo celular en su conjunto encontramos que sólo entre las 6:00 y 8:00 horas parece desarrollarse francamente la mitosis, aunque a través de la acumulación de profases. Durante las demás horas se registra en forma muy consistente un alto porcentaje de interfases, es decir, las células o no se dividen o se dividen poco. La permanencia de las eclulas 
Cuadro $N^{*}$ IA. Evaluación Indices de Fases e Indices Mitáticos Parciales

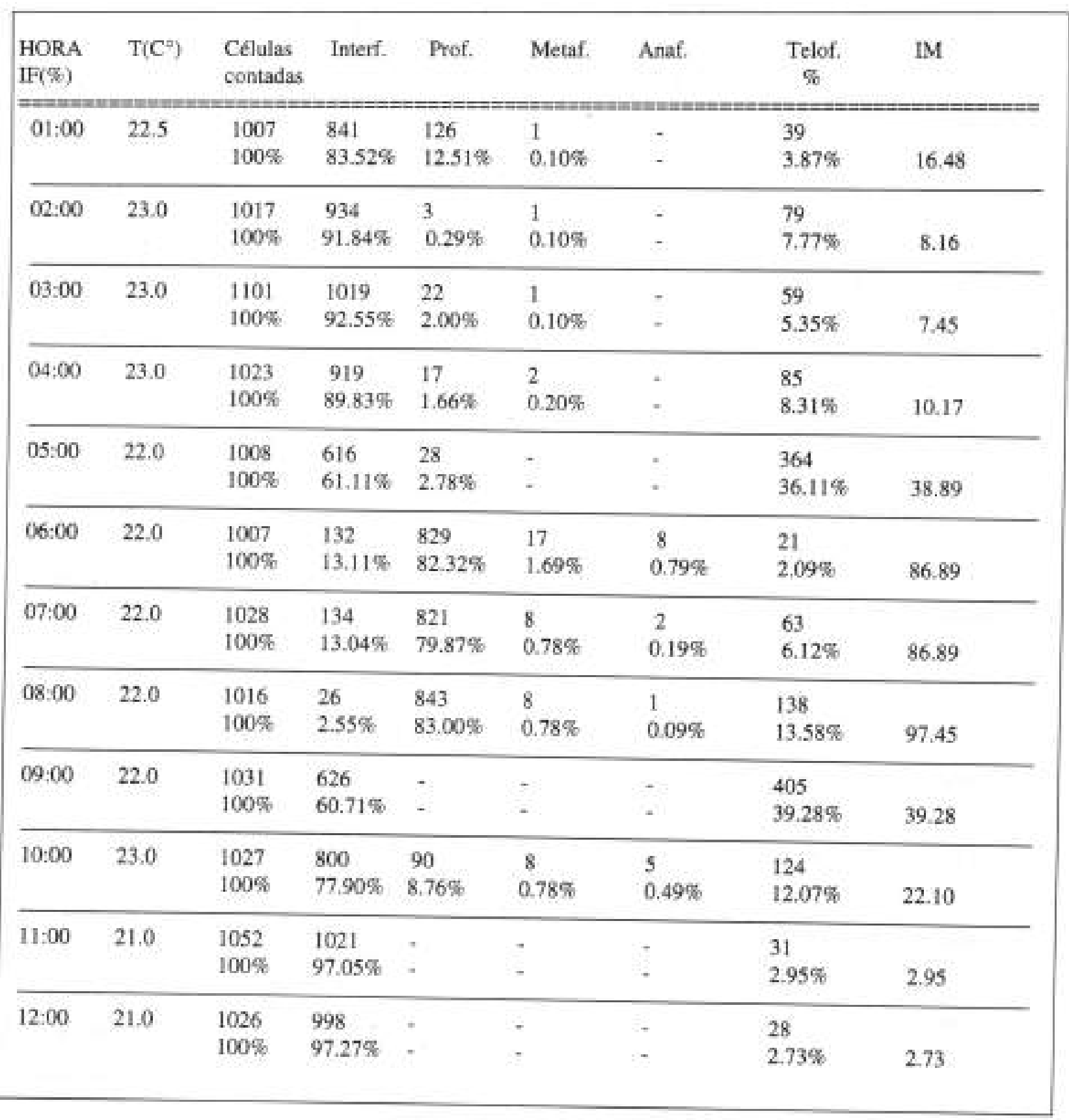


Cuadro $N^{*}$ IB. Evaluación de los Indices de Faser e Indices Mitóticos Parciales

\begin{tabular}{|c|c|c|c|c|c|c|c|c|}
\hline $\begin{array}{l}\text { HORA } \\
\text { IF(\%) }\end{array}$ & $T\left(C^{\circ}\right)$ & $\begin{array}{l}\text { Células } \\
\text { contadas }\end{array}$ & Interf. & Prof. & Metaf. & Anaf. & Telof. & $\begin{array}{r}\text { IM } \\
\text { (\%) }\end{array}$ \\
\hline $13: 00$ & 21.5 & $\begin{array}{r}1014 \\
100 \%\end{array}$ & $\begin{array}{r}926 \\
91.32 \%\end{array}$ & - & $\dot{.}$ & : & $\begin{array}{r}88 \\
8.68 \%\end{array}$ & 8.68 \\
\hline $14: 00$ & 22.0 & $\begin{array}{r}1052 \\
100 \%\end{array}$ & $\begin{array}{r}910 \\
87.00 \%\end{array}$ & - & : & . & $\begin{array}{r}142 \\
13.00 \%\end{array}$ & 13.00 \\
\hline $15: 00$ & 22.5 & $\begin{array}{r}1027 \\
100 \%\end{array}$ & $\begin{array}{r}969 \\
94,35 \text { 的 }\end{array}$ & $\begin{array}{r}22 \\
2.14 \%\end{array}$ & : & - & $\begin{array}{r}36 \\
3.51 \%\end{array}$ & 5.65 \\
\hline $16: 00$ & 23.0 & $\begin{array}{r}1016 \\
100 \%\end{array}$ & $\begin{array}{r}975 \\
95.96 \%\end{array}$ & - & . & . & $\begin{array}{r}41 \\
4.04 \%\end{array}$ & 4.04 \\
\hline $17: 00$ & 23.0 & $\begin{array}{r}1002 \\
100 \%\end{array}$ & $\begin{array}{r}950 \\
94.81 \%\end{array}$ & $\dot{-}$ & : & : & $\begin{array}{r}52 \\
5.19 \%\end{array}$ & 5.19 \\
\hline $18: 00$ & 23,0 & $\begin{array}{r}1022 \\
100 \%\end{array}$ & $\begin{array}{r}950 \\
92.95 \%\end{array}$ & $0.59 \%$ & $0.30 \%$ & : & $\begin{array}{r}63 \\
6.16 \%\end{array}$ & 7.05 \\
\hline $19: 00$ & 23.0 & $\begin{array}{r}1035 \\
100 \%\end{array}$ & $\begin{array}{r}941 \\
90.92 \%\end{array}$ & $\begin{array}{r}27 \\
2.61 \%\end{array}$ & $0.19 \%$ & : & $\begin{array}{r}65 \\
6.28 \%\end{array}$ & 9.08 \\
\hline $20: 00$ & 22.5 & $\begin{array}{r}1021 \\
100 \%\end{array}$ & $\begin{array}{r}936 \\
91.67 \%\end{array}$ & - & $\begin{array}{r}8 \\
0.78 \%\end{array}$ & $\dot{.}$ & $\begin{array}{r}77 \\
7.54 \%\end{array}$ & 8.32 \\
\hline $21: 00$ & 22.0 & $\begin{array}{r}1007 \\
100 \%\end{array}$ & $\begin{array}{r}991 \\
98.41 \%\end{array}$ & - & . & - & $\begin{array}{r}16 \\
1.59 \%\end{array}$ & 1.59 \\
\hline $22: 00$ & 22.0 & $\begin{array}{r}1013 \\
100 \%\end{array}$ & $\begin{array}{r}935 \\
92.30 \%\end{array}$ & $\begin{array}{r}36 \\
3.55 \%\end{array}$ & : & : & $\begin{array}{r}42 \\
4.15 \%\end{array}$ & 7.70 \\
\hline $23: 00$ & 22.5 & $\begin{array}{l}1027 \quad 11 \\
100 \%\end{array}$ & $\begin{array}{r}11 \quad 859 \\
10.81 \%\end{array}$ & $\begin{array}{r}26 \\
83.64 \%\end{array}$ & $\begin{array}{r}5 \\
2.53 \%\end{array}$ & $\begin{array}{r}26 \\
0.49 \%\end{array}$ & $2.53 \%$ & 89.19 \\
\hline $24: 00$ & 22.5 & $\begin{array}{r}1018 \\
100 \%\end{array}$ & $\begin{array}{r}782 \\
76.82 \%\end{array}$ & $\begin{array}{r}99 \\
9.72 \%\end{array}$ & $\begin{array}{r}8 \\
0.79 \%\end{array}$ & : & $\begin{array}{r}129 \\
12.67\end{array}$ & 23.18 \\
\hline
\end{tabular}


en una u otra fase del ciclo sugiere el bloqueo o insuficiencia de la síntesis protéica en la fase anterior por lo que que el material genético, luego de duplicarse en la interfase, no necesariamente se va a distribuir entre células hijas.

\section{DISCUSION}

El procesamiento citológico de O. tuberosa $\mathrm{Mol}$. consistente en la determinación de los f́ndices de fases (IF), indices mitóticos (IM) y secuencia del ciclo celular en condiciones de laboratorio permitió establecer IM elevados y próximos al $100 \%$ (IM $\left.\omega_{00}=86.89 \%, \mathrm{IM}_{\omega \infty}=97.45 \%\right)$, to que coincide con lo reportado por Talledo y Escobar (1995) para $U$. tuberosum. Respecto al desarrollo del ciclo celular, el análisis de los cuadros $1 \mathrm{~A}, 1 \mathrm{~B}$ y 2 permite evidenciar que durante el periodo que corresponde a la mitosis el porcentaje de metafases y anafases supera el $1 \%$ sólo a las 6:00 am. y durante el resto de horas (excepto las 23:00) está por debajo de esta cifra. El porcentaje de profases y telofases se mantiene elevado durante parte importante de la misma, es más las células parecen detenidas en estas fases. Si tomamos en cuenta que una serie de trabajos reportan la posibilidad de alterar en especies modelo la secuencia normal de la división celular en sus diferentes fases (Brooks, 1977; Del Campo, 1988: Epifánova, 1973; Prescott, 1976; Nagl, 1970, 1978), que Prokofieva-Belgovskaya (1959, 1960) observó la aparición đe células binucleadas y mitosis con diplocromosomas en muestras de papa y que la variación de las condiciones ambientales puede ser la causa de la alteración del flujo normal de la mitosis, encontraremos que en oca puede estar produciéndose un fenómeno probable en este tipo de muestras, aunque poco conocido. Nuestros resultados sugierea fuertemente: a) el desarrollo de ciclos celulares incompletos (ciclos que no transcurren por una $o$ varias de las fases normales) y b). El bloqueo en profases y del paso de telofase hacia la

Cuadro $N^{*}$ 2. Deterninacion de los IF e IM totales y de la duración del ciclo celular.

\begin{tabular}{|lllllll|}
\hline Estado & Total & Interf. & Prof. & Metaf. & Anaf. & Telof. \\
\hline Frecuencia & 24,597 & 18,442 & 3,828 & 93 & 21 & 2,213 \\
\hline IF (\%) & 100 & 74.98 & 15.56 & 0.38 & 0.08 & 9.00 \\
\hline IM (\%) & 25.02 & & $(15.56+0.38+0.08+9.00)$ & \\
\hline $\begin{array}{l}\text { Duracion Hirs } \\
\text { asumiendo un } \\
\text { valor medio } \\
\text { para un ciclo } \\
\text { completo=5Hrs }\end{array}$ & $5: 00$ & $(3.75)$ & $(0.78)$ & $(0.02)$ & $(0.01)$ & $(0.45)$ \\
\hline
\end{tabular}

interfase (es decir, curso defectuoso de las fases intermedias $y$ finales de la mitosis) en parte importante de la población celular estudiada.

La scparación temporal de la celula del ciclo normal de división parece haber condicionado la prolongación del mismo en parte importante de la población celular estudiada. La aparente duración de 6 horas de la mitosis, mencionada en RESULTADOS para esta especie, no corresponde a la de un ciclo normal (que suele ocupar entre $45^{\prime}$ y 75 ), como fué el caso del ciclo de división celular del olluco, que ocupó 60 minutos (Talledo y Escobar, 1995). El ciclo normal de división se estaría produciendo sólo en parte de esta población y su duración sería de 75 minutos (Gráfico 1).

El estudio citológico de 50 especies de Oxalis permitió a Brucher (1969) sugerir un número base de $\mathrm{n}(\mathrm{x})=7$ para las especies de este género y múltiplos de 7 como número cromosómico. Durante el presente estudio celular se pudo establecer en base al contaje de cromosomas en las metafases de no menos de 15 células sin ningún pre-tratamiento que el número cromosómico de esta accesión de aOca* es $2 \mathrm{n}=14$ (Foto 3, Fig. 1). Este resultado sugiere que $\mathrm{n}=\mathrm{x}=7$ y coincide con lo propuesto por Heitz (1927) y Bricher (1969).

El bloqueo de la mitosis o parte de ella no impide la reproducción de los cromosomas; a su vez, la separación de los cromosomas y la formación de aúcleos hijos no son predeterminantes para la citotomía. Como consecuencia, en los ciclos siguientes a partir de estas mitosis incompletas (es decir, que no presentan algunas de sus fases normales) será pxsible observar metafases con complementos cromosómicos duplicados o con diplocromosomas (Mezia, 1963; Epifánoya, 1973). Esto podría explicar la diversidad de reportes respecto al 
número de cromosomas de la oca, asi como la gran dificultad, sefialada por una serie de autores (Gibbs, 1978; Medina, 1994) para evidenciar cariotipos en cromosomas mitóticos. El análisis de la distribución de los números de cromosomas de Oxalis reportados hasta la fecha corrobora esta apreciación. En el cuadro 3 y fig. 3 podemos observar que los principales «picosa de números cromosómicos $(2 n)$ para especies de este género corresponden a 14 y 28 , que para 10 de ellas se han reportado números diferentes (en 8 casos múltiples de 7)

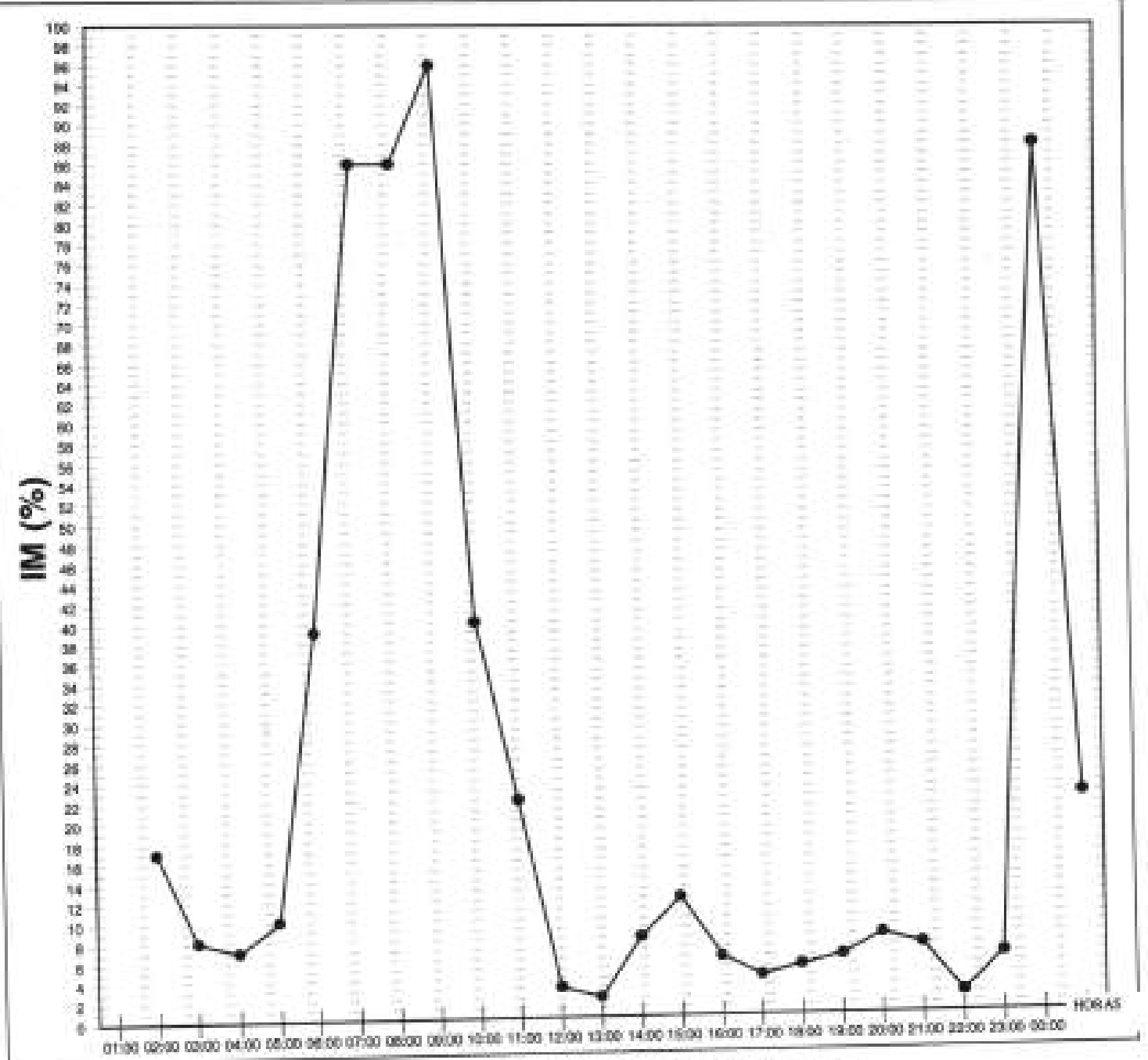

Gráfico 1. Secieencia del ciclo celalar en Oxalis tuberosa

y que en las especies que presentan poliploidia intraespecifica ésta se produce con un número base $\mathrm{x}=7$. Lo5 trabajos que sugieren númetos cromosómicos diferentes a máltiplos de 7 por lo general sugieren cifras muy próximas a éstos: 20,$22 ; 30 ; 48 ; 64,66$.

La inhibición parcial de los procesos mitóticos en estas celulas puede reflejar el desarrollo normal de estos organismos o haber sido desencadenada por el traslado de los mismos a un hábitat diferente. En cualquier caso es preciso tomarla en cuenta tanto para el desarrollo de trabajos de campo como para la conservación y reproducciónin vitro.

Entre las plantas de papa obtenidas por cultivo de tejidos se observa una gran variabilidad fenotipica (Karp et al, 1982; Shepard, 1980), la misma que es inberente a todos los regenerantes obtenidos por cultivo de tejidos. Se ha reportado que parte de esta variabilidad puede deberse a variaciones del numero y estructura cromosómicos y que estas variaciones son resultado de la manifestación de selectividad del medio respecto a las células del explante primario. Respecto a las células de oca parece estar ocurriendo algo similar. Es evidente que una población como la descrita presentará inevitahlemente varias lineas celulares diferentes, por lo que la variabilidad somaclonal puede ser inherente a la muestra o, en el mejor de los easos, a algún tipo de muestra sacado fuera de su entorno natural.

\section{CONCLUSIONES}

El estudio del ciclo celular en $O$. tuberosa Mol. procedente de la ciudad de Huancayo, PERU, nos ha permitido esclarecer los siguientes aspectos, hasta ahora desconocidos, del desarrollo de este cultivo: 


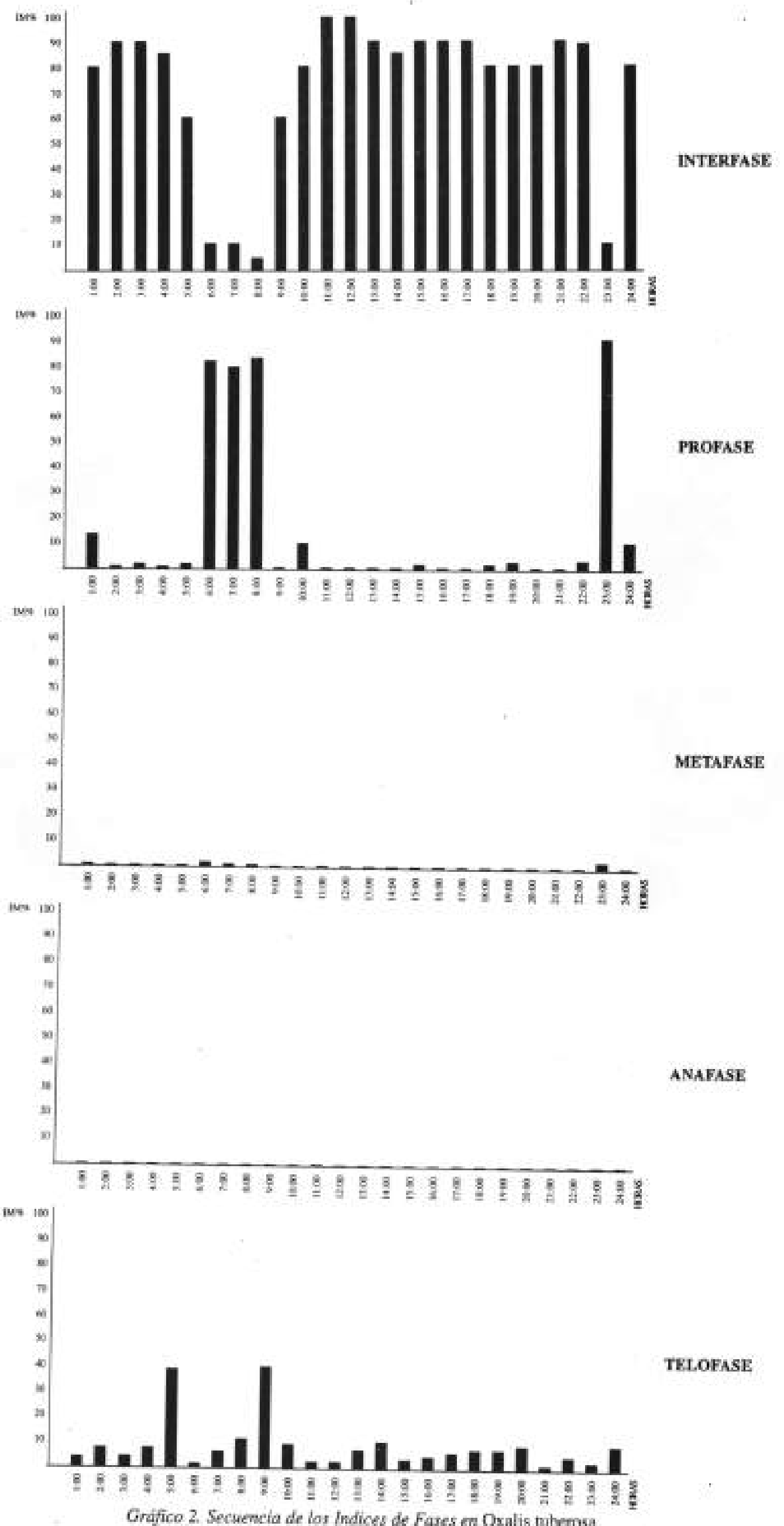

Gráfico 2. Secuencia de los Indices de Fases en Oxalis tuberosa 


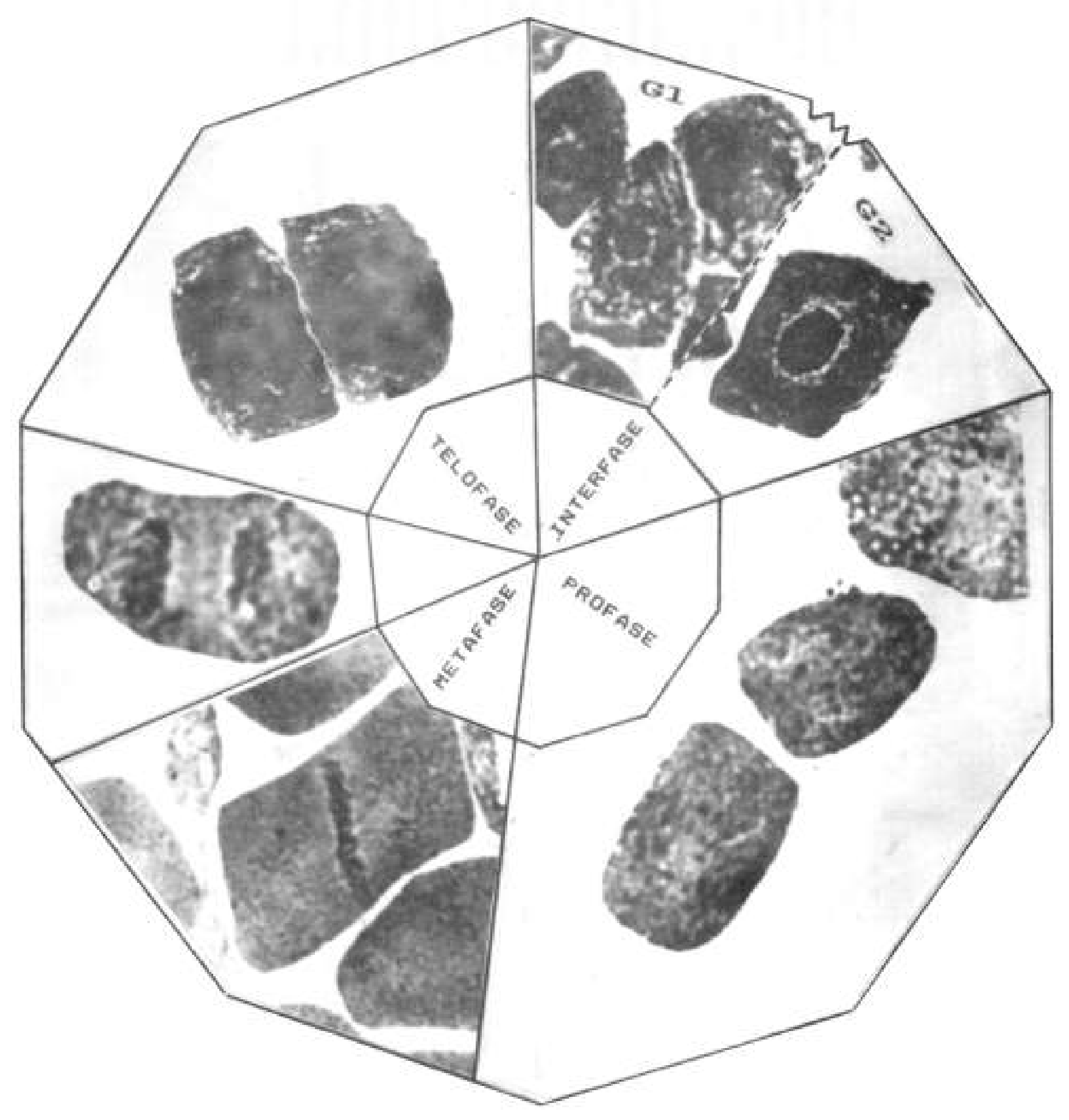

Foro 2. Fases del ciclo celular en Oxalis uberosa 


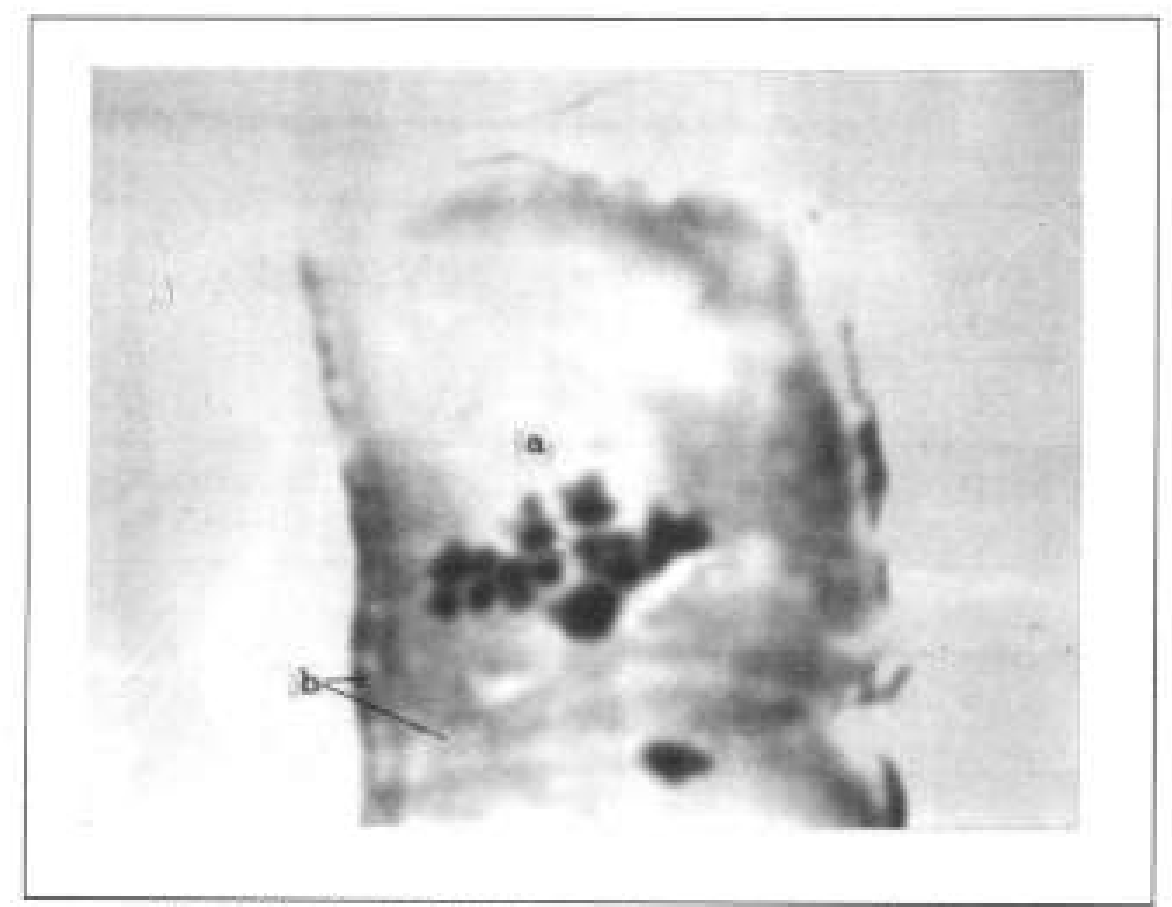

Foto 3. Mercfase de Oxalis taberosa sin pre-fratamiento $(2 n=14) ; a)$ Cremosamas, b) Membrana celitalar.

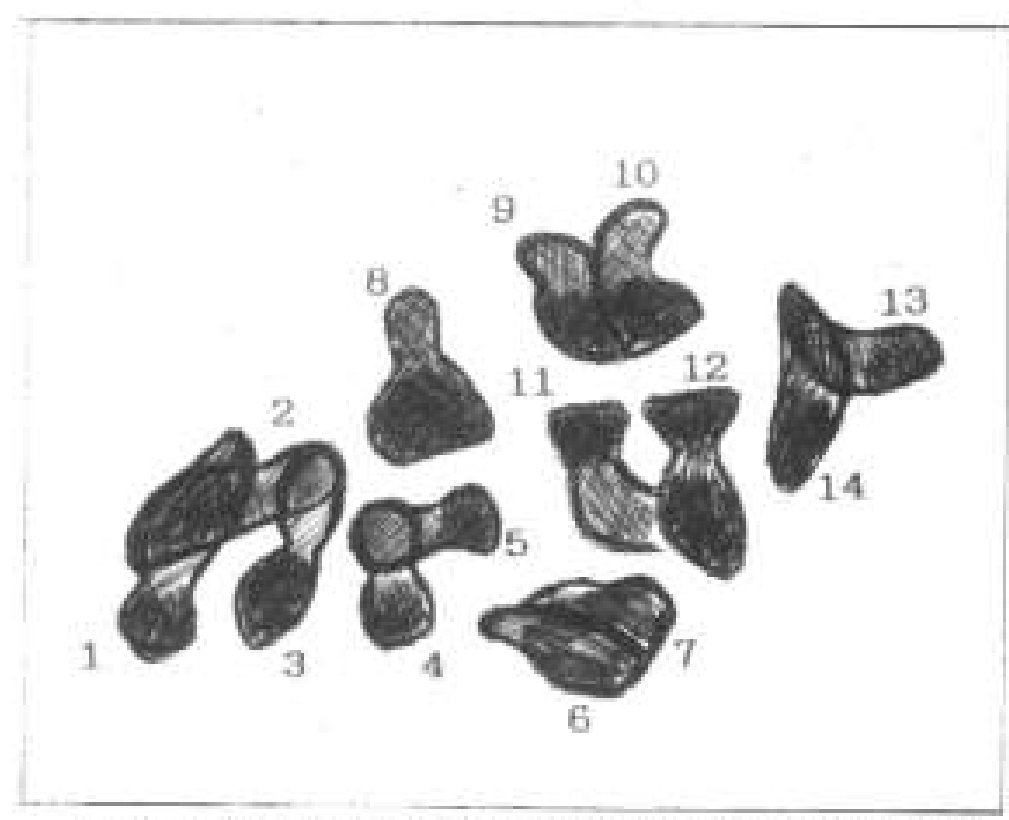

Figura f. Esquemanisaciain de los 14 cromosomas obsenvados en la metafase de la Foro 3.

1. En algunas accesiones de esta especie se producen ciclos celulares incompletos, es decir, que prescntan procesos mitóticos en los cuales se ha(n) reducido una o varias de las fases normales de la mitosis.

2. Estos ciclos celulares incompletos parccen deberse al bloqueo parcial en profase y en telofase, las causas de este bloqueo autin no estaín claras.

3. Los fenómenos mencionados en las conclusiones (1) y (2) condicionàn una alteración general de la división de estas células, aumentando la duración del ciclo hasta llegar a valores evidentemente anormales.
4. El periodo de sintesis de la interfase, y la interfase en general, parecen desarrollarse normalmente, por lo que la reduplicación del material genético se estaria produciendo en forma normal.

5. La reduplicación normal de la cromatina $y$ la división anormal de la misma serían los factores condicionantes de la variabilidad encontrada para el número de cromosomas de esta especie por una serie de autores.

6. El periodo comprendido entre las 6:00 y 8:00 horas parece ser el más aparente para la toma de muestras celulares de "Oca* para estudios cariotípicos. 
Cradro $N^{\prime \prime} 3$ Dismbuciein de Numerus Cromotémicos en Oxatis $\mathrm{L}$

\begin{tabular}{|c|c|c|}
\hline $\begin{array}{l}\text { Número de } \\
\text { especies }\end{array}$ & $2 n$ & Autores \\
\hline 2 & 10 & $\begin{array}{l}\text { Heitz, 1927; Yamashita, 1935; Marks, 1956; } \\
\text { Borgmann, 1964. }\end{array}$ \\
\hline 3 & 12 & Marks, 1956, 1957; $\left(^{* *}\right)$ \\
\hline 20 & 14 & $\begin{array}{l}\text { Winge, 1925; Heitz, 1926, 1927; Yamashita, 1935; } \\
\text { Warburg, 1938; Marks, 1956; Diers, } 1961\end{array}$ \\
\hline 2 & 16 & Eiten, 1963; $(* *)$ \\
\hline 3 & 18 & $\begin{array}{l}\text { Warburg, 1938; Marks, 1956; Diers, 1961; } \\
\text { Eiten, } 1963\end{array}$ \\
\hline 1 & 22 & $\begin{array}{l}\text { Nakajima, 1936; Love, Love, D., 1966; Hara, } 1952 \\
\text { Marks, 1956; Skalinska et. al., 1959; Sorsa V., } 1962 \\
\text { Gadella, Kliphuis, 1963; Hara, Kurosawa, } 1965\end{array}$ \\
\hline 1 & 24 & $\begin{array}{l}\text { Wulff, 1937; Rutland, 1941: Heiser, Whitaker, } 1948 \\
\text { Polya, 1949; Skalínska et. al.,1959; Eiten, 1963; } \\
\text { Gadella, Kliphuis, } 1966\end{array}$ \\
\hline 9 & 28 & $\begin{array}{l}\text { Heite, 1927; Yamashita, 1935; Warburg, 1938; } \\
\text { Marks, 1956; Matew, 1958; Borgmann, } 1964 \text {. }\end{array}$ \\
\hline 3 & 30 & $\begin{array}{l}\text { Heitz, 1927; Yamashita, 1935; Marks, } 1956 \\
\text { Sharma A.K., Chatterii T., } 1960 .\end{array}$ \\
\hline 2 & 35 & $\begin{array}{l}\text { Vignoli, 1935. 1937; Yamashita, 1935; } \\
\text { Matsuura Suzo, 1935; Heiser, Whitaker, } 1948 .\end{array}$ \\
\hline 2 & 40 & Marks, $1956 ;(* *)$ \\
\hline 3. & 42 & $\begin{array}{l}\text { Heitz, 1927; Marks, 1956; Sharma A.K., } \\
\text { Chatterji T., 1960; (**). }\end{array}$ \\
\hline 2 & 48 & $\begin{array}{l}\text { Marks, 1956; Mathen, 1958; Mathew, 1958; } \\
\text { Sharma A.K., Chatterji T., 1960; Diers, } 1961\end{array}$ \\
\hline 1 & 56 & Marks, 1956; Mathew, 1958; (**) \\
\hline 1 & 64 & Kostov, 1935; De Azkue, 1990; Medina, 1993: \\
\hline 1 & 80 & Heitz, 1927; (**) \\
\hline 5 & $\mathrm{PI}(*)$ & Varios; $(* *)$ \\
\hline 10 & $\begin{array}{l}\text { Diferentes } \\
\text { números }\end{array}$ & Varios; $(* *)$ \\
\hline 73 & & Total de Especies Estudiadas \\
\hline
\end{tabular}




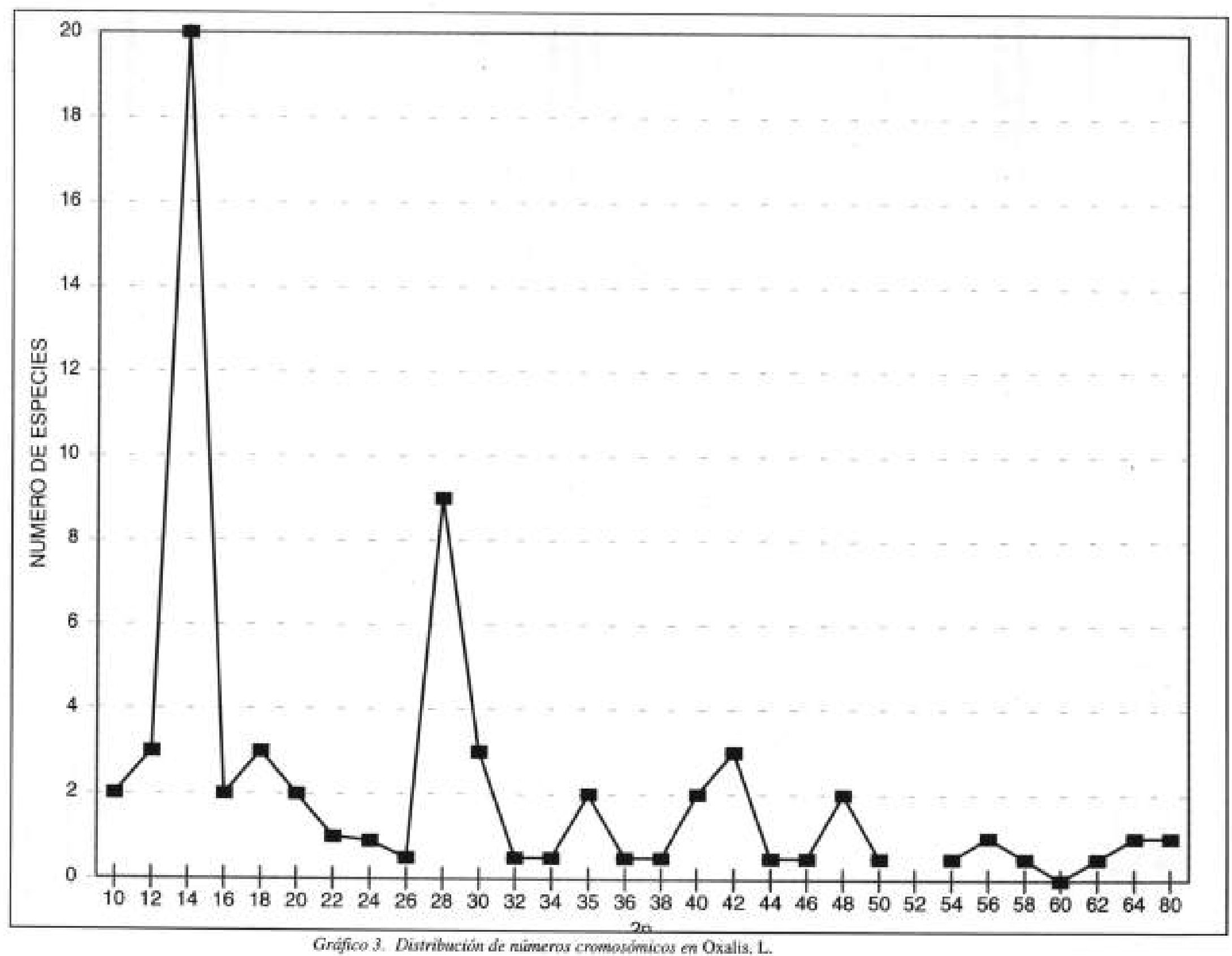


7. Nuestros resultados evidencian que el número cromosómico de la muestra estudiada de $O$. tuberosa Mol. es $2 n=2 x=14$, siendo su número base $x=7$.

8. Los números cromosómicos diferentes a $2 \mathrm{n}=14$, pero múltiplos de $x=7$, reportados por una serie de autores, al igual que la variabilidad somaclonal observada en algunas formas de *Oeaw introducidas in vitro, se habrian producido en base a los procesos mencionados en las conclusiones (1), (2), (4) y (5).

9. Los fenómenos evidenciados, descritos y analizados en el presente trabajo indican claramente que el analisis cariotípico de las muestras es imprescindible tanto para su caracterización en condiciones naturales como para la introducción de las mismas in vitro.

\section{AGRADECIMIENTOS}

Los autores queremos expresar nuestro reconocimiento al Programa Colaborativo COTESU - CIPy a la Universidad Ricardo Palma, Rectorado, Facultad de ciencias Biologicas y Departamento Académico de Ciencias), sin cuyo apoyo no hubiera sido posible desarrollar el presente trabajo.

\section{REFERENCIAS BIBLIOGRAFICAS}

BENNETT, M.D. 1972. Nuclear DNA content and minimum generation time in herbaceous plants, Proc. Roy. Soc. Landon B. (I8I): 109-135.

BOLKHOVSKIKH, Z.; GRIF, V.; MATVEJEVA, T.; ZAKHARYEVA, O. 1969. Chromosome numbers of flowering plants. Leningrad. Nauka. 926 pp.

BORGMANN, E 1964. Anteil der Polyploiden in der Flora des Bismarcksgebirges von Ostneuguinea_Zeitschr. Bot. 52 (2): 118-173.

BRODSKY, V.Y ; URIVAYEVA, LV. 1981. (Poliploidía celular. Proliferación y diferenciación), Ed. Naúka, Moscui. (En ruso).

BROOKS, R.F. 1977. Continuous protein synthesis is required to maintain the probubility of entry into $\mathrm{S}$ phase. Cell.12; 311-317.

BROCHER, H, 1969. Poliploidfa en especies sudamericanas de Oxalis, - Boletín Soc. Venezol. Clenc. Natur. 28 (115/116): 145-178.

CARDENAS, M. Y HAWKES, J.G. 1948. Número de cromosomas en algunas plantas nativas cultivadas por los indios de los Andes- Revista de Agricultura (Cachabamba) $5(4): 30-32$.

CIRF, 1982:Descriptores de Oca-Conscjo Internacional de Recursos Fitogenéticos Programa del CIRF en América Latina.
CARDENAS, M. 1969, Manual de plantas económicas de Bolivia. Cochabamba: Imprenta Icthus. pp 16-86.

DE AZKUE, D. Y MARTíNEZ, A. 1983. The chromosome complements of Shrubby Oxalis species from South America.- Pl.Syst.Evol.141: 187-197.

DE AZKUE, D. Y MARTÍNEZ, A, 1990. Chromosome number of the Oxalis tuberosa alliance (Oxalidaceae).Pl. Syst. Evol. 169, 25-29.

DEL CAMPO, A., 1988. Biología del Ciclo de División celular. Maracaibo, Venezuela. Univ, de Zulia, Fac. Experimental de Ciencias. 151 pp.

DEL RIO, C.A. 1990, Análisis de la variación iscenzimática de Oxalis nuberosa Molina «Oca» y su distribución geográfica- Tesis Lic,en Biologia. Universidad Ricardo Palma, Facultad de Ciencias Biologicas. 61 p. Lima-Peri.

DIERS, L. 1961. Der Anteil an Polyploden in den Vegetationsgurtein der Westkordillere Perus. Zeitschr. Bot., 49, 5:437-488.

EITEN, G. 1963. Taxonomy and regional variation of Oxalis sect Corniculatae 1. Introduction, keys and sinopsis of the species. Amer. Midl. Nat, 69:257-309.

EPIFANOVA, O.I, 1973. (Esrudio de los mecanismos de regulación del ciclo celular por medio de inhibidores de la transcripción y la translación. En: El ciclo celular). Ed. Naúka. Moscui. PP. 72-103. (En ruso).

ESTRADA, R. 1994, Reunión POA, Proyecto R2. Cuzco, (Comunicación oral).

FIÓDOROV, A.A 1974. Chromosome Numbers of Flowering Plants:- Leningrad. Academia de Ciencias. URSS. (Ed. Otto Kocltz Sci. Pub.).

GADELLA, T.W.J.; KLIPHUIS E, 1963. Chromosome numbers of flowering plants in the Netherlands. Acra Bot. Neerlandica, 12, 2:195-230,

GADELLA, T.W.J; KLIPHUIS E. 1966. Chromosome numbers of flowering plants in the Netherlands. Acta BOL Neerlandica, 13, 3:432-433.

GERSHOFF, S.N. 1987. Nutritional evaluation of three underexploited Andean tubers: Oxalis tuberosa, Ullacus tuberosus and Tropaeolum tuberosum,- Econ. Bot. 4I: 503-519.

GIBBS, PE; MARSHALL, D. Y BRUNTON, D, 1978. Studies on the cytology of Oxalis tuberosa and Tropaeolum tuberosume NotRoy.Gard. Edinburgh. 37:215-220. 
HARA, H. 1952. Contributions to the study of varitions in the Japanese plants closely related to those of Europe or North America. Part I. Jour. Fac. Sci. Tokyo Imp. Univ, Sect. 3 Bot. 6, 1-3:29:96

HARA,H.; KUROSAWA, S. 1965. Cytotaxonomical studies on Japono-Himalayan elements. Jour. Japanese Bot., 40, 2:4-8.

HEISER,C.B; WHITAKER, T.W. 1948. Chromosome number,polyploidy and growth habit in California weeds. Amer, Joir. Bot. 35, 3: 179-186.

HEIIZ E 1927. Ueber multiple und aberrante Chromosomenzahlen.Abhandl. Naturwiss. Vereins Hamburg, 21, 3-4:47-57.

IVANOV, V.B. 1978. (Contenido de ADN nuclear y velocidad de desarrollo de las plantas). Ontogenes. T. 9. PP. 39-53.

JARDIN BOTANICO DE CORDOBA (ESPAÑA). 1992. Cultivos marginados: Otra perspectiva de 1492. Colección FAO: Producción Agrícola y protección vegetal. $\mathrm{N}^{*} 26.339 \mathrm{p}$.

KARP, A.; NELSON, R.S.; THOMAS, E.; BRIGHT, S.WJ, 1982.Chromosome variation in protoplastderived potato plants. Theor.Appl.Genet. 63: 265-272.

KOSTOFF, D. DOGADKINA, N Y TICHONOV, A. 1935. Chromosomenumbers of certain angiosperm plants.(Nicotiana, Petunia, Oxalis, Secale and Punica).Compt. Rend. Acad Sci. URSS. 3(9):401-404.

LEON, J. 1964, Plantas Alimenticias Andinas,- Instituto Interamericano de Ciéncias Agricolas. Boletin Técnico $N^{*}$ 6. Lima.Perú.

LOVE, A.; LOVE, D. 1966. Cytotaxonomy of the alpine vascular plants of mount Washington. Univ. of Colorado Studies, Ser. Biol. 24:1-74.

MARKS, G.E. 1956. Chromosome numbers in the genus Oxalis L. - New Phytologist 55, 120-129.

MARKS, G.E 1957. The eytology of Oxalis dispar (Brown). Chromosoma, 8, 6:650-670

MATHEW, PM 1958. Cytology of Oxalidaceae. Cytologia, 23, 2;200-210

MATSUURA, H, SUTO, T, 1935. Contributions to the idiogram study in phanerogamous plants. I. Joar. FaC. Sci. Hokkaido Imp. Univ., Ser 5, Bot., 5, 5:33-75.

MEDINA, H.T. 1994. Contaje cromosómico de la Oca (Oralis tuberosa Mol.). - Tesis ... Ing* Agr. Universidad Nacional del Centro del Perú. 47 pp. Huancayo-Peri.
MEZIA, D. 1963. (La mitosis y la fisiologia de la división celular). Ed. Mir, Moscú. 345 pp. (En ruso).

MOSTACERO, J. Y MEJIA, F, 1993. Taxonomia de fanerogamas peruanas. CONCYTEC. Lima-Perú.

NAGL, W. 1970. Differential inhibition by actinomycin $\mathrm{D}$ and histone $\mathrm{F}_{1}$ of mitosis and endomitosis in Allimm carinatum. 2. Pflanzenphysiol. 63: 316-326.

NAG, W, 1978. Endopolyploidy and polyteny in differentiation and evolution. Amsterdum: North-Holland.

NAKAJIMA, G. 1936. Chromosome numbers in some crops and wild Angiosperms, Japanese Jour, Genefics, $12,6: 211-218$.

NATIONAL RESEARCH COUNCII., 1989. Lost crops of the Incas:Little-Known plants of the Andes with Promise for Worldwide Cultivation, National Academy Press, Washington, D.C. 415 pP.

PRESCOTT, D.M. 1976. Reproduction of eukaryotic cells. N.Y.; L. Acad. Press.

PROKOFIEVA-BEL GOVSKAYAA.A. 1959 (Interacciones del núcleo y el citoplasma ea celulas de almacenamiento de almidón en papa). Citologia. T, 1. PP, 257269. (En ruso).

PROKOFIEVA-BELGOVSKAYA, A.A. 1960, (Ciclo nuclear ydiferenciación de las células somaticas. En: Problemas de la Citología y la Fisiología General). Ed. Ac. de CC. de la URSS, Moscú, Leningrada. PP. 215-253, (En ruso). POLYA, L. 1949. Chromosome numbers of some Hungarian plants. Acta Geobot. Hungarica, 6,2:124-137.

RUTLAND, J.P. 1941. The Merton catalogue. A list of chromosome numbers of British plants. New Phytol. 40 , 3:210-214

SHARMA A.K., CHATTERUT, T. 1960. Cytological studies on three species de Oxalis. Caryologia. 13, 3:755-765.

SHEPARD, J.F. 1980 Matant selection and plant regeneration from potato mesophyll protoplasts. In: Emergent techniques for the genetic improvement of crops. Minneapolis. PP. 185-219.

SKALINSKA, M ; CZAPIK, R:PIOTROWICZ, M, et al. 1959. Further studies in chromosome numbers of Polish angiosperms (Dicotyledons). Acta Soc. Bot, Polon., 28 , 3:487-529.

SMrTH, PM. $19 \pi \%$ Minor crops, in: Simmonds, N.W.(ed.) Evolution of Crop Plants. London \& New York. 
SORSA, V. 1962. Chromosomenzahlen Finnischer Kormophyten. I. Ann. Acad. Sei. Fennica, Ser. A, IV, Biol., 58:1-14.

STEBBINS, G. L. 1970, Variation and evolution in plants: Progress during the past twenty years. PP. 173 208. In: Essays in Evolution and Genetics in Honor of Th. Dobzhansky. N.Y, USA.

TAL,LEDO, D., ESCOBAR, C, 1995. El ciclo celular en vegetales: su estudio, importancia y aplicaciones. Biotempo 2:

TALLEDO, D; ESCOBAR, C; ALLEMAN, V, 1993. Introducción al análisis cromosómico en vegetales. Universidad Ricardo Palma, Fac. de Ciencias Biologicas. Lima. $141 \mathrm{pp}$.

TURKOV, V.D:; GUZHOV, YU.L.; SHELEPINA, G.A. KISHMARIA.YA.SH:; KOMETIANI, D.G. 1988. (Las investigaciones cromosómicas de vegetales en el Fitomejoramiento, la Ingeniería Celular y el Monitoreo Genético). Atlas-Manual 64 pp. Ed. UDN. Moscú, URSS. (En ruso).
VAN'T HOFF, J; SPARROW, A M. 1963. A relationship between DNA content, nuclear volume and minimum mitotic cycle time. Proc. Nat. Acad. Sci. USA. 49:897-902.

VELASCO, E. 1994, Reunión POA. Proyecto R2. Cuzco. (Comunicación oral), COTESU-CIP.

VIGNOLI, L. 1935. Ricerche preliminari di cytologia sull' Oxalis cernua Thunb. Nuovo Giorn. Bof, Ital, 42, 4:668-669.

VIGNOLI, L. 1937. Fenomeni niproduttivi di Oxatis cemua Thunb. Lavori Ist. Bot. Palermo, 8-5-30.

WARBURG, E.F, 1938. Taxonomy and relationship in the Geraniales in the light of their cytology. II. New Physol., 37, 3:189-210.

WINGE, O. 1925. Contributions to the knowledge of chromosome numbers in plants. Cellule, 35:303-324

WULF, HD. 1937. Chromosomenstudien an der schleswigholsteinischen Angiospermen-Flora. I. Ber. Deutsch.Bot.Ges, 55, 4:262-269.

YAMASHITA, K. 1935. Zytologische Studien an Oxalis. I. Japanese. Genetics, II, 1:36. 